\title{
High-Resolution Depth-Encoding PET Detector Module with Prismatoid Light-Guide Array
}

\author{
Andy LaBella ${ }^{1}$, Xinjie $\mathrm{Cao}^{2}$, Eric Petersen ${ }^{1}$, Rick Lubinsky ${ }^{3}$, Anat Biegon ${ }^{3}$, Wei Zhao ${ }^{3}$, and Amir H. Goldan ${ }^{3}$ \\ ${ }^{I}$ Department of Biomedical Engineering, College of Engineering and Applied Sciences, Stony Brook University, Stony Brook, New \\ York; ${ }^{2}$ Department of Electrical and Computer Engineering, College of Engineering and Applied Sciences, Stony Brook University, \\ Stony Brook, New York; and ${ }^{3}$ Department of Radiology, Renaissance School of Medicine, Stony Brook University, Stony Brook, \\ New York
}

Depth-encoding detectors with single-ended readout provide a practical, cost-effective approach for constructing high-resolution and high-sensitivity PET scanners. However, the current iteration of such detectors uses a uniform glass light-guide to achieve depth encoding, resulting in nonuniform performance throughout the detector array due to suboptimal intercrystal light sharing. We introduce Prism-PET, a single-ended-readout PET detector module with a segmented light-guide composed of an array of prismatoids that introduce enhanced, deterministic light sharing. Methods: High-resolution PET detector modules were fabricated with singleended readout of polished multicrystal lutetium yttrium orthosilicate scintillator arrays directly coupled 4-to- 1 and 9-to- 1 to arrays of $3 \times$ $3 \mathrm{~mm}$ silicon photomultiplier pixels. Each scintillator array was coupled at the nonreadout side to a light-guide (one 4-to-1 module with a uniform glass light-guide, one 4-to-1 Prism-PET module, and one 9-to-1 Prism-PET module) to introduce intercrystal light sharing, which closely mimics the behavior of dual-ended readout, with the additional benefit of improved crystal identification. Flood histogram data were acquired using a $3-\mathrm{MBq}^{22} \mathrm{Na}$ source to characterize crystal identification and energy resolution. Lead collimation was used to acquire data at specific depths to determine depth-of-interaction (DOI) resolution. Results: The flood histogram measurements showed excellent and uniform crystal separation throughout the Prism-PET modules, whereas the uniform glass light-guide module had performance degradation at the edges and corners. A DOI resolution of $5.0 \mathrm{~mm}$ full width at half maximum (FWHM) and an energy resolution of $13 \%$ FWHM were obtained in the uniform glass light-guide module. By comparison, the 4-to-1 coupled Prism-PET module achieved a DOI resolution of $2.5 \mathrm{~mm}$ FWHM and an energy resolution of $9 \%$ FWHM. Conclusion: PET scanners based on our Prism-PET modules with segmented prismatoid lightguide arrays can achieve high and uniform spatial resolution (9-to-1 coupling with $\sim 1$-mm crystals), high sensitivity (20-mm-thick detectors and intercrystal Compton scatter recovery), good energy and timing resolutions (using polished crystals and after applying DOI correction), and compact size (depth encoding eliminates parallax error and permits smaller ring-diameter).

\footnotetext{
Received Dec. 6, 2019; revision accepted Feb. 10, 2020.

For correspondence or reprints contact: Amir H. Goldan, Renaissance School of Medicine, Stony Brook University, 101 Nicolls Rd., Stony Brook, NY, 11794

E-mail: amirhossein.goldan@stonybrookmedicine.edu

Published online Feb. 28, 2020.

COPYRIGHT @ 2020 by the Society of Nuclear Medicine and Molecular Imaging.
}

Key Words: instrumentation; optical; PET; DOI; light-guide; PET; prism

J Nucl Med 2020; 61:1528-1533

DOI: 10.2967/jnumed.119.239343

$\mathbf{M}$ olecular imaging with PET is a powerful technique used primarily for diagnosis, treatment selection, treatment monitoring, and research in cancer (1) and neuropsychiatric disorders (2). Despite its high molecular specificity, quantitative nature, and clinical availability, PET has not been able to achieve its full potential as the go-to molecular imaging modality, largely because of its relatively poor spatial resolution, currently on the order of 3-6 $\mathrm{mm}(3,4)$. With this kind of spatial resolution, it is not possible to measure target density in small nodules or in many human and rodent brain regions relevant to disease etiology and pathophysiology.

Depth-encoding PET detector modules have been developed to mitigate parallax error (mispositioning of the line of response) for long scintillator crystals (5). These modules enable small-diameter PET detector rings with a reduced component cost per ring, large solid-angle coverage for increased sensitivity, and a reduced contribution from annihilation $\gamma$-ray acollinearity on spatial resolution when using crystals with a small cross-sectional area $(4,6)$. In addition, depth-of-interaction (DOI) information can be used to deconvolve optical photon transport in long crystals, thus improving timing resolution $(7,8)$. Depth-encoding detectors based on dual-ended readout achieve the best continuous DOI resolution of less than $2 \mathrm{~mm}(9,10)$. High-resolution PET systems such as the mammography-dedicated Clear-PEM have been developed using dual-ended DOI readout detectors (11), but these systems are too costly to be commercialized because of the large number of readout electronics compared with standard single-ended-readout PET scanners. A recently developed high-resolution variant of these detectors shows relatively poor energy and timing resolutions because the crystal-readout interface includes the use of glass light-guides, which are required to achieve accurate crystal identification (12). Alternative single-ended-readout detector modules to obtain DOI information have been proposed, such as multilayer phoswich blocks $(13,14)$, retroreflectors for modules with monolithic scintillators (15), and other custom reflector designs $(16,17)$. However, in all these designs, tradeoffs exist among depth encoding, cost, scintillator-to-readout coupling ratio, crystal identification accuracy, energy resolution, and timing resolution. To 
mitigate these tradeoffs, an ideal depth-encoding detector module is one with single-ended readout in which the crystal array is coupled directly to silicon photomultiplier (SiPM) pixels, without any intermediate glass light-guide, to minimize sharing of downward-traveling scintillation photons (i.e., photons traveling toward the SiPM) across multiple pixels and retain a good timing resolution. In addition, upward-traveling photons (i.e., photons traveling toward the light-guide), which do not contribute to the timing information, should be redirected via $180^{\circ}$ bending of their paths toward the nearest neighboring SiPMs to retain good energy and DOI resolution and mimic the behavior of dual-ended depthencoding readout detectors.

Detector modules consisting of depolished multicrystal scintillator arrays coupled 4-to-1 to SiPM pixels on one side and a uniform glass light-guide on the opposite side (Fig. 1A) have recently been investigated in efforts to develop a practical and costeffective high-resolution time-of-flight PET scanner, as well as achieve continuous DOI localization using single-ended readout $(8,18,19)$. In these detector modules, an energy weighted-average method is used for crystal identification to achieve energy and DOI resolutions of $9 \%$ and $3 \mathrm{~mm}$ in full width at half maximum $(\mathrm{FWHM})$, respectively, using $1.53 \times 1.53 \times 15 \mathrm{~mm}$ crystals and $3 \times 3 \mathrm{~mm}$ SiPM pixels (8). However, these arrays suffer from poor crystal identification along their edges and corners due to the lack of light-sharing neighbors (19), an issue that must be addressed since the edge and corner pixels comprise $75 \%$ and $44 \%$ of $4 \times 4$ and $8 \times 8$ SiPM readout chips, respectively. Also, intercrystal light sharing is inefficient when using a uniform glass light-guide since many upward-traveling photons are reflected back into the primary column and the rest are isotropically shared with a gaussian intensity distribution among neighbors. The problem with isotropic light sharing is the distribution of lowintensity signal across many SiPMs (Fig. 1A), the integrity of which

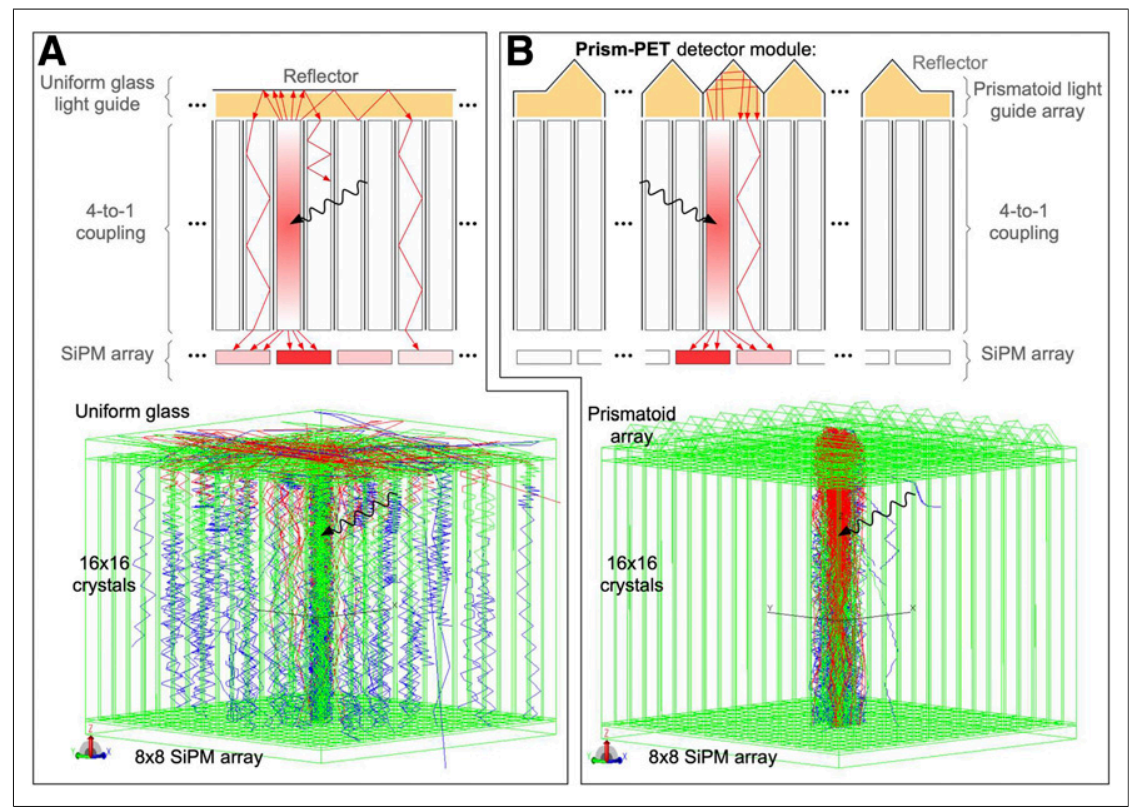

FIGURE 1. Comparison of light-sharing scheme between uniform glass light-guide module (A) and Prism-PET (B) as simulated in TracePro (20). Light is uniformly distributed via light sharing in uniform light-guide, whereas prismatoid light-guide array in Prism-PET confines light sharing to crystals coupled to the same prismatoid and enhances intercrystal light-sharing ratios. will be severely affected by dark counts, resulting in degraded energy and DOI resolutions.

We introduce the concept of Prism-PET (20), a single-endedreadout depth-encoding detector module that uses a specialized pattern of segmented prismatoid light-guides (Fig. 1B). Our Prism-PET detector modules have several key features. First, we use 3 distinct prismatoid designs (center, edge, and corner prismatoids) to mitigate edge and corner artifacts and thus achieve uniform crystal identification performance. Second, we confine intercrystal light sharing to only the nearest SiPM neighbors to create a deterministic and anisotropic intercrystal light-sharing pattern and maximize the signal-to-background ratio on those SiPMs to improve both energy and DOI resolutions. The segmentation pattern is the key feature that improves crystal identification by decoupling adjacent crystals that would otherwise have similar readout patterns; thus, the shape of each prismatoid is interchangeable (cuboids, pyramids, wedges, prisms, cupolas, frusta, and others). In this study, right triangular prisms were used. Third, the right triangular prisms enhance intercrystal light-sharing ratios, thus improving both crystal identification and DOI resolution. When optical photons enter the hypotenuse of the right triangular prisms, they undergo $180^{\circ}$ deviation, efficiently guiding them to neighboring crystals, which are coupled to different readout pixels because of the offset crystal-to-prism coupling scheme with respect to crystal-to-pixel coupling (Fig. 2). Using experimental measurements, we demonstrate the advantages of our design in terms of crystal identification, energy resolution, and DOI resolution, including how Prism-PET enables up to 9-to-1 crystal-to-readout coupling, which can be used to substantially improve spatial resolution without increasing the number of readout channels (Figs. 2C and 2D).

\section{MATERIALS AND METHODS}

\section{Device Fabrication}

We fabricated 3 different PET detector modules for comparison. One consisted of a $16 \times 16$ array of $1.4 \times 1.4 \times 20 \mathrm{~mm}$ lutetium yttrium orthosilicate (LYSO) crystals coupled 4-to- 1 on one side to an $8 \times 8 \mathrm{SiPM}$ readout array and on the opposite (radiation-receiving) side to a uniform glass light-guide, similar to modules previously studied in the literature $(8,21)$. The second module consisted of the same crystal and readout geometry, but we interchanged the single uniform glass light-guide with a prismatoid lightguide array that has a unique design and layout of prisms at the corner, edge, and center of the detector module to optimize light-sharing patterns (Figs. 2A and 2B). The third module used $0.96 \times 0.96 \times 20 \mathrm{~mm}$ LYSO crystals to achieve 9-to-1 coupling while keeping the prismatoid light guide array identical to the second module (Figs. 2C and 2D). In both Prism-PET modules, scintillator crystals are coupled to readout pixels and prismatoids in equal ratios. The coupling scheme of the prisms is offset from that of the readout pixels, such that each crystal is coupled only to other crystals belonging to different 


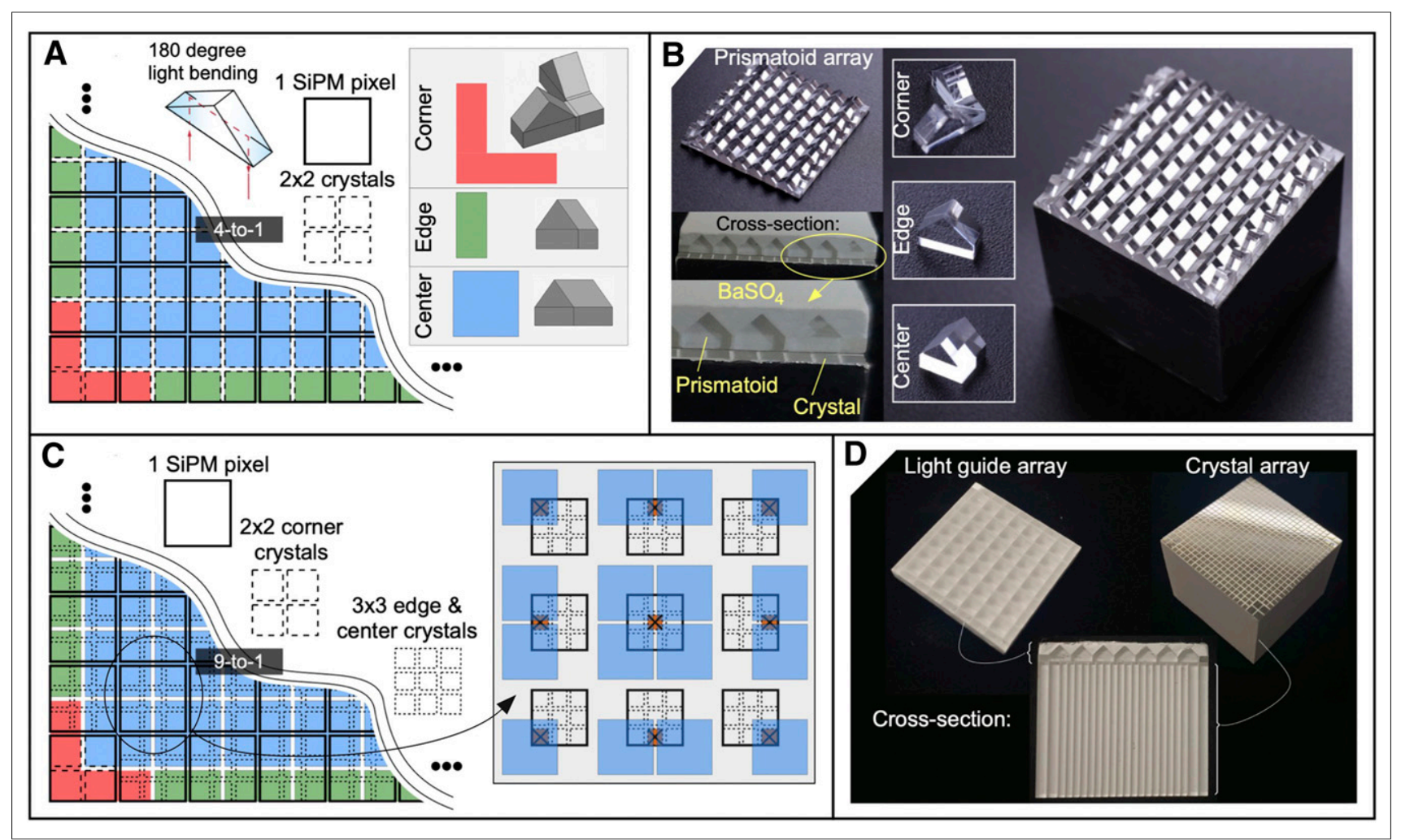

FIGURE 2. Concepts and fabrication of Prism-PET detector modules. (A and B) Schematic diagram and fabrication of 4-to-1 coupled Prism-PET module. ( $C$ and D) Schematic diagram and fabrication of 9-to-1 coupled Prism-PET module. Inset in $C$ shows unique readout pattern of each of the 9 crystals that are coupled to the same SiPM pixel.

readout pixels (Fig. 2A). When optical photons enter the prismatoids after $\gamma$-ray interactions in the crystals, they are efficiently redirected to neighboring crystals because of the right triangular prism geometry, enhancing the light-sharing ratio between pixels (Fig. 1). The geometry of each prismatoid is position-dependent to decouple adjacent crystals along the edges and corners that would otherwise have similar readout patterns in order to optimize crystal separation.

Additionally, because the coupling scheme confines intercrystal light sharing to be between neighboring SiPMs that enhance crystal identification, one can match the index of refraction between the scintillator columns, prisms, and coupling adhesive to further enhance light sharing and consequently improve DOI resolution and crystal identification. All prisms were fabricated using SF10 optical glass with an index of refraction of 1.77 (instead of BK7 optical glass with an index of refraction of 1.53 , which is the material for the uniform glass light-guide) and coupled to the scintillator arrays using Norland Optical Adhesive 170 (Norland Products, Inc.) with an index of refraction of 1.7. Barium sulfate $\left(\mathrm{BaSO}_{4}\right)$ is used as the reflector material between the crystals and prisms because of its high spatial performance, which does not degrade energy or timing resolutions (22). SiPM saturation effects, which have been known to positively skew energy resolution and negatively impact DOI resolution, were not accounted for in this study and will be explored in detail in future work (23).

\section{Detector Readout}

The detector modules in this study consisted of LYSO crystal arrays fabricated at X-Lum, coupled (either 4-to-1 or 9-to-1) to $8 \times 8$ arrays of SiPMs (S13361-3050AE-08; Hamamatsu). Data were acquired using TOFPET2 application-specific integrated circuits and a FEB/D (version 2) readout board from PETsys Electronics. Flood data were acquired by uniformly exposing the modules with a $3-\mathrm{MBq}$ ${ }^{22} \mathrm{Na}$ point source $(5-\mathrm{mm}$ active diameter). In total, $10,000,000$ events from the 4-to- 1 module and 22,500,000 events from the 9-to-1 module (to acquire an equal number of events per crystal) were used for flood histogram generation.

\section{Electronic Tagging Setup}

We experimentally measured the DOI performance of our modules on a per-crystal basis using an approach similar to one previously described (18). The modules were exposed to a ${ }^{22} \mathrm{Na}$ source at 5 different crystal depths $(2,6,10,14$, and $18 \mathrm{~mm})$ using lead collimation. The source was placed in a lead cylinder with a 1-mm pinhole. The pinhole was aligned with the DOI-aligned module on one side and a single $1.4 \times 1.4 \times 20 \mathrm{~mm}$ crystal on a reference module on the other side. Coincidence events between the 2 modules were used to reject scatter events and accept only events along the intended line of response. The histograms for the DOI-estimation parameter (18), $w$, were calculated and plotted for all crystals. The gaussian-shaped $w$ histograms were then converted to DOI space using linear regression to determine the slope between $w$ and the ground-truth DOI (Supplemental Fig. 1; supplemental materials are available at http://jnm.snmjournals.org). DOI resolution is depth-dependent and equal to the FWHM of the gaussian histograms. Overall crystal-specific DOI resolution was calculated as the average of the DOI resolutions across the measured depths (18). A typical center crystal from each module was used to calculate the DOI resolutions of each module. 


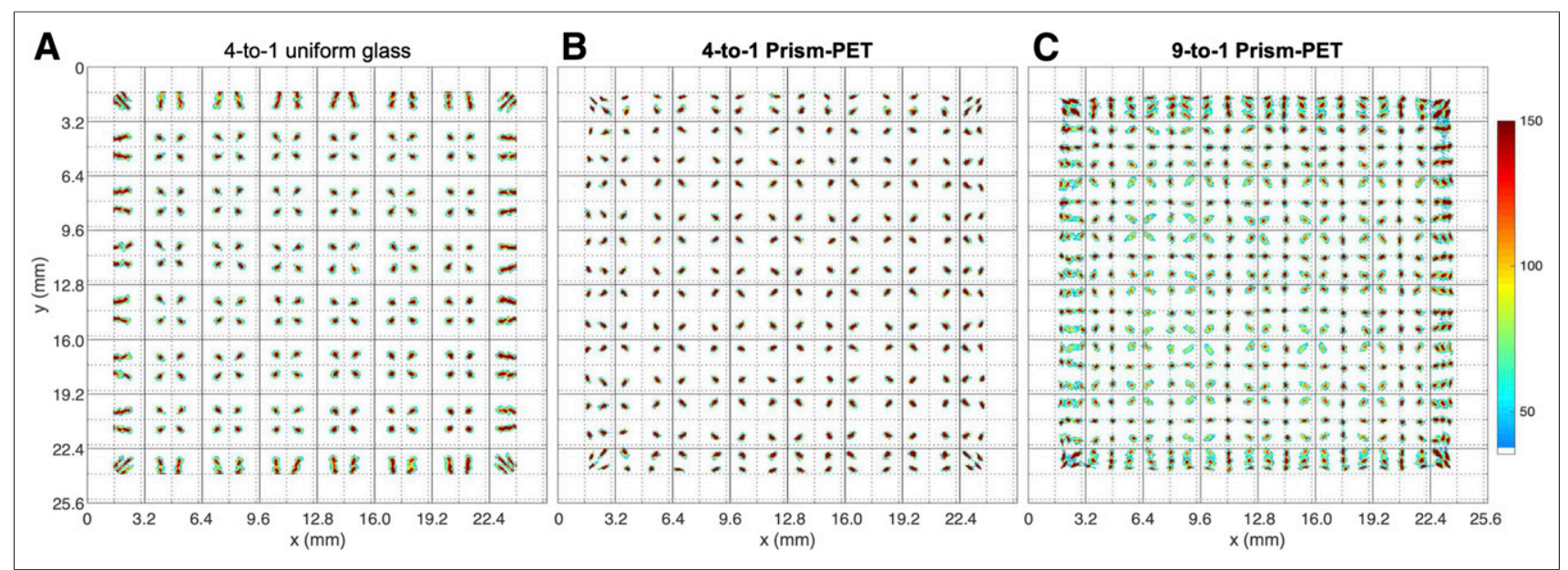

FIGURE 3. Experimental results for crystal identification. Shown are flood histogram data for 4-to-1 coupled module with flat uniform glass lightguide (A), 4-to-1 coupled Prism-PET module (B), and 9-to-1 coupled Prism-PET module (C).

\section{RESULTS}

We characterized the spatial performance of our fabricated modules using flood histogram measurements. The uniform glass light-guide module suffers from edge and corner artifacts, resulting in poor position-dependent crystal identification (Fig. 3A). Prism-PET enables excellent crystal separation throughout the entire detector array, without edge or corner artifacts (Figs. $3 \mathrm{~B}$ and $3 \mathrm{C}$ ), which (to our knowledge) has not previously been achieved in a 4-to-1 coupled detector module with single-ended time-of-flight DOI readout $(8,19,21)$. We showed similar results with the 9-to-1 coupled Prism-PET module (Fig. 3C), demonstrating homogeneous submillimeter crystal separation in a time-of-flight DOI PET detector module with $3 \times 3 \mathrm{~mm} \mathrm{SiPM}$ pixels. Plotting 1-dimensional event positioning histograms (in the $x$-direction) confirms that Prism-PET has uniform crystal separation performance at the center, edges, and corners (Fig. 4A). Prism-PET also achieves 14\% and $16 \%$ energy resolution without DOI correction in the 4-to-1 and 9-to-1 coupled modules, respectively, whereas the uniform lightguide module achieves $20 \%$ energy resolution (Fig. 4B).

We also experimentally measured the DOI performance for a single center crystal in each module (Fig. 5). The measured DOI resolution for the glass light-guide had a FWHM of $5 \mathrm{~mm}$, showing strong agreement with previously reported results (19). The Prism-PET modules achieved 2.5-mm-FWHM DOI localization, the best resolution ever reported using single-ended readout. Increased depth-dependence of the $w$ parameter is due to a controlled and deterministic light-sharing pattern within the prismatoids, increased light transfer from scintillators to lightguides due to matched refractive indices, and enhanced deviation of the upwardtraveling optical photon path by $180^{\circ}$ due to the right triangular prism geometry, all of which enhance light sharing between crystals coupled to the same prismatoid. DOI information can be used to improve both timing and energy resolution, the former by deconvolving depth-specific photon transport inside the scintillator and the latter by constructing depth-specific photopeaks $(8,18)$. We achieved $9 \%$ and $10 \%$ energy resolution in the 4-to- 1 and 9-to-1 coupled Prism-PET modules, respectively, and $13 \%$ energy resolution with the uniform light-guide after applying DOIbased correction (Fig. 4B). Note that the DOI and energy resolution values will slightly change for better and worse, respectively, after implementing SiPM saturation correction (23); as a result, the values reported in this current study are more indicative of the relative performance of PrismPET compared with the uniform light-guide module than the absolute performance in practice.
FIGURE 4. (A) One-dimensional gaussian histograms showing crystal separation in $x$-direction from corner, edge, and center readout pixel for all 3 modules. (B) Filtered energy spectrums with (black) and without (colored) DOI correction from center crystals in all 3 modules. 


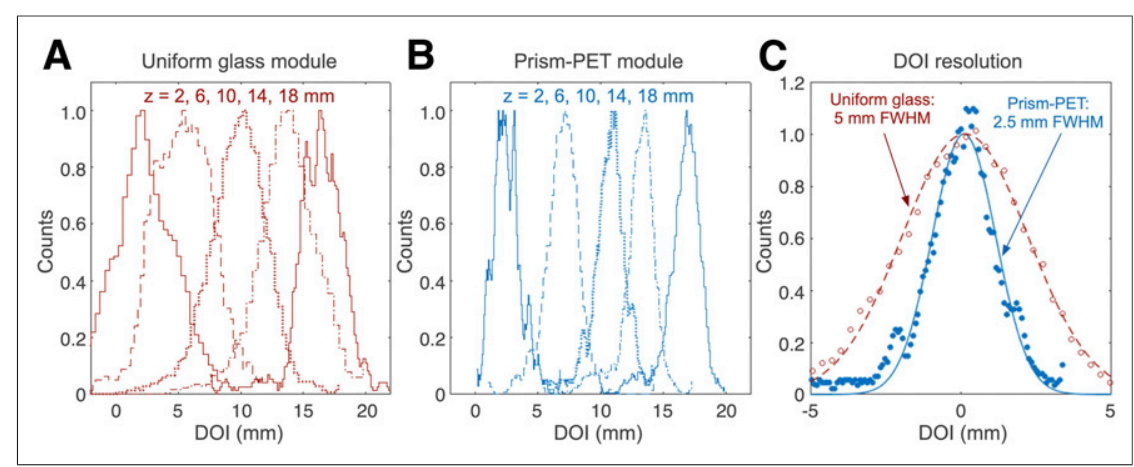

FIGURE 5. Experimental results for DOI resolution. (A and B) DOI histograms (calculated from the measured $w$ variable) for $5 z$ positions of the electronic tagging setup in center crystals of 4-to1 coupled detector modules with uniform glass (A) and prismatoid light-guides (B). (C) Comparison of DOI resolution based on light-guide used. Prism-PET detector module achieves 2-fold improvement in DOI resolution over uniform glass light-guide.

\section{DISCUSSION}

We have developed a cost-effective and practical method for achieving high spatial and DOI resolution in multicrystal singleended-readout detector modules without introducing edge or corner artifacts. Our technology can be used to enable depth encoding in clinical whole-body and total-body PET scanners (24) without increasing cost (the prismatoid light-guide array comprises less than $10 \%$ of the total cost of each Prism-PET module) or power consumption while potentially improving spatial resolution (via 9-to- 1 coupling of, for example, $2 \times 2 \times 20 \mathrm{~mm}$ crystals to $6 \times 6 \mathrm{~mm}$ readout pixels), sensitivity (via intercrystal Compton scatter recovery), and timing resolution (via DOI correction of timing jitter). For small-ring-diameter brain imaging, the 9-to-1 coupling ratio enables submillimeter spatial resolution, and extending the axial field-of-view to about double that of wholebody PET scanners enables the same geometric sensitivity gain as for the EXPLORER total-body PET scanner (Supplemental Fig. 2; Supplemental Note 1) (8,24-27). In addition, having 2.5-mm DOI resolution greatly mitigates parallax error and potentially enables achieving an approximately 100-ps coincidence time resolution via DOI correction (8), which would enable even higher sensitivity and spatial resolution (25-27). These benefits yield a practical, cost-effective, and power-efficient approach to achieving both high spatial resolution and high sensitivity at a relatively low dose for quantitative in vivo functional and molecular imaging of many human body organs that have not been resolvable with existing PET scanners, including important structures of the brain such as the raphe nuclei, cholinergic basal forebrain nuclei, locus coeruleus, and hypothalamic nuclei, all of which are thought to play crucial roles in basic physiology and in the pathophysiology of common neurodegenerative and psychiatric disorders (27-31). The ability to visualize and quantitate these and similar targets has the potential to revolutionize molecular imaging in both the clinical and the research arenas, providing hitherto unavailable tools for early diagnosis and basic research in oncology and brain disorders.

Another potential advantage of Prism-PET is the ability to more accurately identify the initial interaction site of Compton scatter events, further improving spatial resolution and sensitivity (Supplemental Note 2; Supplemental Figs. 3-4). Traditionally, Compton detection has been performed using multiple detector layers, but a recent paper outlined the criteria for localizing and decomposing Compton interactions using single-ended readout, citing high-resolution DOI readout as a key feature for Compton scatter recovery (32). A uniform light-guide is not optimal for this task because the SiPM pattern of individual events is random, whereas our Prism-PET modules create a deterministic light-sharing pattern regardless of the interaction location inside the primary scintillator column (Fig. 1; Supplemental Fig. 4). Most notably, Prism-PET enables the decomposition of side-by-side scattered photon and recoil electron eventswhich are the scattering events that are most probable and most difficult to analyze-into their constituent energies, spatial location, and DOI (Supplemental Note 2). Compton scatter recovery is especially critical to retain high sensitivity in detector modules with small scintillator crystals, since the scattered photon is more likely to be absorbed in a crystal different from that of the primary interaction site as crystal size decreases (33).

\section{CONCLUSION}

We have developed, fabricated, and characterized our proposed Prism-PET detector module, which is a true single-ended equivalent of a dual-ended depth-encoding readout using efficient $180^{\circ}$ light-bending reflectors for enhanced light sharing. We achieved 2.5-mm-FWHM DOI resolution and up to 9-to-1 scintillator-to-SiPM coupling for high spatial resolution while directly coupling the crystal array to the SiPM pixels to minimize light leakage and retain high photon detection efficiency, which is required for good timing resolution. The top-side reflector comprises an optimized pattern of segmented prismatoid light-guides for efficient redirection of scintillation photon paths from the primary crystal to selected nearest-neighboring SiPMs, thus closely mimicking the operation of dual-ended-readout detectors. This creates an anisotropic and deterministic pattern of signal that can be used to decompose side-by-side Compton scattering events into their constituent energy and DOI information for the purpose of scatter recovery. Thus, we can expect to achieve high and uniform spatial resolution (9-to-1 coupling of $\sim 1$-mm crystals; absence of edge and corner artifacts due to enhanced light sharing; reduced spatial blur due to Compton-scattered photons via scatter recovery), high sensitivity (20-mm-thick detectors and intercrystal Compton scatter recovery), and good energy and timing resolutions (especially after applying DOI correction) in compact systems (DOI encoding eliminates parallax error and permits smaller ring-diameter). With these unique combinations of features, cost-effective and compact time-of-flight DOI-Compton PET scanners based on Prism-PET modules could be developed for small-animal and human organspecific functional and molecular imaging.

\section{DISCLOSURE}

Financial support was received from the National Institutes of Health (R21 EB024849). No other potential conflict of interest relevant to this article was reported. 


\section{KEY POINTS}

QUESTION: Can we develop a high-resolution ( 1- to 2-mm crystals) single-ended-readout (low-cost) PET detector module with simultaneous depth-encoding and time-of-flight capabilities?

PERTINENT FINDINGS: We developed a segmented light-guide made of an array of right triangular prisms that can be coupled to the radiation-receiving end of a PET module. Our light-guide enables up to 9-to-1 scintillator-to-readout coupling ( 1 -mm spatial resolution when using standard $3 \times 3 \mathrm{~mm}$ readout pixels) and 2.5-mm-FWHM depth-of-interaction resolution (similar to the performance of dual-ended-readout modules) without sacrificing sensitivity or coincidence time resolution.

IMPLICATIONS FOR PATIENT CARE: Cost-effective high-resolution and high-sensitivity PET systems can be built with our detector module design, including preclinical, human organdedicated, and total-body scanners.

\section{ACKNOWLEDGMENT}

We gratefully acknowledge PETsys Electronics, SA, Portugal, for scientific discussions.

\section{REFERENCES}

1. Mankoff DA, Farwell MD, Clark AS, Pryma DA. Making molecular imaging a clinical tool for precision oncology: a review. JAMA Oncol. 2017;3:695701.

2. Slifstein M, Abi-Dargham A. Recent developments in molecular brain imaging of neuropsychiatric disorders. Semin Nucl Med. 2017;47:54-63.

3. van Sluis J, De Jong J, Schaar J, et al. Performance characteristics of the digital Biograph Vision PET/CT system. J Nucl Med. 2019;60:1031-1036.

4. Moses WW. Fundamental limits of spatial resolution in PET. Nucl Instrum Methods Phys Res A. 2011;648(suppl):S236-S240.

5. Miyaoka RS, Lewellen TK, Yu H, McDaniel DL. Design of a depth of interaction (DOI) PET detector module. IEEE Trans Nucl Sci. 1998;45:1069-1073.

6. Stickel JR, Cherry SR. High-resolution PET detector design: modelling components of intrinsic spatial resolution. Phys Med Biol. 2005;50:179-195.

7. Seifert $S$, Schaart DR. Improving the time resolution of TOF-PET detectors by double-sided readout. IEEE Trans Nucl Sci. 2015;62:3-11.

8. Pizzichemi M, Polesel A, Stringhini G, et al. On light sharing TOF-PET modules with $3 \mathrm{~mm}$ depth of interaction and 157 ps FWHM coincidence time resolution. Phys Med Biol. 2019;64:155008.

9. Yang Y, Wu Y, Qi J, et al. A prototype PET scanner with DOI-encoding detectors. J Nucl Med. 2008;49:1132-1140.

10. Kuang Z, Wang X, Fu X, et al. Dual-ended readout small animal PET detector by using $0.5 \mathrm{~mm}$ pixelated LYSO crystal arrays and SiPMs. Nucl Instrum Methods Phys Res A. 2019;917:1-8.

11. Abreu MC, Aguiar JD, Almeida FG, et al. Design and evaluation of the clear-PEM scanner for positron emission mammography. IEEE Trans Nucl Sci. 2006;53:71-77.
12. Du J, Yang Y, Bai X, et al. Characterization of large-area SiPM array for PET applications. IEEE Trans Nucl Sci. 2016;63:8-16.

13. Schmand M, Eriksson L, Casey ME, et al. Performance results of a new DOI detector block for a high resolution PET-LSO research tomograph HRRT. IEEE Trans Nucl Sci. 1998;45:3000-3006.

14. Seidel J, Vaquero JJ, Siegel S, et al. Depth identification accuracy of a three layer phoswich PET detector module. IEEE Trans Nucl Sci. 1999;46:485-490.

15. Gonzalez-Montoro A, Aguilar A, Canizares G, et al. Performance study of a large monolithic LYSO PET detector with accurate photon DOI using retroreflector layers. IEEE Trans Radiat Plasma Med Sci. 2017;1:229-237.

16. Ito M, Lee MS, Lee JS. Continuous depth-of-interaction measurement in a single-layer pixelated crystal array using a single-ended readout. Phys Med Biol. 2013;58:1269-1282.

17. Kuang Z, Yang Q, Wang X, et al. A depth-encoding PET detector that uses light sharing and single-ended readout with silicon photomultipliers. Phys Med Biol. 2018;63:045009.

18. Pizzichemi M, Stringhini G, Niknejad T, et al. A new method for depth of interaction determination in PET detectors. Phys Med Biol. 2016;61:4679-4698.

19. Niknejad T, Pizzichemi M, Stringhini G, et al. Development of high-resolution detector module with depth of interaction identification for positron emission tomography. Nucl Instrum Methods Phys Res A. 2017;845:684-688.

20. LaBella A, Zhao W, Lubinsky R, Goldan AH. Prismatoid light guide array for enhanced gamma ray localization in PET: a Monte Carlo simulation study of scintillation photon transport. Phys Med Biol. May 15, 2020 [Epub ahead of print]. https://doi.org/10.1088/1361-6560/ab9373.

21. Stringhini G, Pizzichemi M, Ghezzi A, et al. Development of a high resolution module for PET scanners. J Instrum. 2017;12:C02073.

22. Kuang Z, Wang X, Li C, et al. Performance of a high-resolution depth encoding PET detector using barium sulfate reflector. Phys Med Biol. 2017;62:59455958.

23. Otte AN, Barral J, Dolgoshein B, et al. A test of silicon photomultipliers as readout for PET. Nucl Instrum Methods Phys Res A. 2005;545:705-715.

24. Badawi RD, Shi H, Hu P, et al. First human imaging studies with the Explorer total-body PET scanner. J Nucl Med. 2019;60:299-303.

25. Surti S. Update on time-of-flight PET imaging. J Nucl Med. 2015;56:98-105.

26. Reddin JS, Scheuermann JS, Bharkhada D, et al. Performance evaluation of the SiPM-based Siemens Biograph Vision PET/CT system. In: Conference Record of the 2018 IEEE Nuclear Science Symposium and Medical Imaging Conference. Piscataway, NJ: IEEE; 2018:1-5.

27. Gong K, Majewski S, Kinahan PE, et al. Designing a compact high performance brain PET scanner: simulation study. Phys Med Biol. 2016;61:3681-3697.

28. Hornung JP. The human raphe nuclei and the serotonergic system. J Chem Neuroanat. 2003;26:331-343.

29. Mufson EJ, Ginsberg SD, Ikonomovic MD, DeKosky ST. Human cholinergic basal forebrain: chemoanatomy and neurologic dysfunction. J Chem Neuroanat. 2003;26:233-242.

30. Betts MJ, Kirilina E, Otaduy MCG, et al. Locus coeruleus imaging as a biomarker for noradrenergic dysfunction in neurodegenerative diseases. Brain. 2019;142:2558-2571.

31. Barbosa DA, de Oliveira-Souza R, Santo FM, et al. The hypothalamus at the crossroads of psychopathology and neurosurgery. Neurosurg Focus. 2017;43:E15.

32. Makek M, Bosnar D, Pavelić L. Scintillator pixel detectors for measurement of Compton scattering. Condens Matter. 2019;4:24.

33. Hsu DF, Freese DL, Innes DR, Levin CS. Intercrystal scatter studies for a $1 \mathrm{~mm}^{3}$ resolution clinical PET system prototype. Phys Med Biol. 2019;64:095024. 